\title{
Estudo funcional e morfológico da hipotermia sistêmica ou tópica no fígado de cães $^{1}$
}

\author{
Functional and morphological study of the local and systemic hypothermia on \\ dog's liver.
}

\author{
Venilton José Siqueira², Murched Omar Taha ${ }^{3}$, Djalma José Fagundes ${ }^{4}$, Paulo de Oliveira Gomes ${ }^{4}$, Yara Juliano ${ }^{5}$, Renata \\ Marcon zanelatto Bruzzadelli ${ }^{6}$, Lucélia Rita Galdino Caputto ${ }^{7}$
}

1. Tese de Doutorado, Disciplina de Técnica Operatória e Cirurgia Experimental Universidade Federal de São Paulo - Escola Paulista de Medicina.

2. Prof. da Disciplina de Técnica Operatória e Anestesiologia da Faculdade de Medicina Veterinária da Universidade José do Rosário Vellano - UNIFENAS.

3. Prof. Adjunto Afiliadodo Departamento de Cirurgia - Serviço de Técnica Operatória e Cirurgia Experimental UNIFESPEPM

4. Prof. Adjunto do Departamento de Cirurgia - Serviço de Técnica Operatória e Cirurgia Experimental UNIFESP-EPM

5. Prof. Adjunto do Departamento de Medicina Preventiva - Disciplina bioestatística UNIFESP-EPM

6. Prof ${ }^{a}$. da Disciplina de Patologia Clínica Faculdade de Medicina Veterinária da UNIFENAS.

7. Prof ${ }^{\text {a }}$ da disciplina de Patologia e Medicina Legal da Faculdade de Ciências Médicas da UNIFENAS

\begin{abstract}
RESUMO
Objetivo: Estudar as alterações morfo-funcionais hepáticas produzidas por dois tipos de hipotermia; a sistêmica ou tópica (localizada). Métodos: 20 cães foram distribuídos em dois grupos: G I (n=10) submetido a hipotermia tópica e G II (n=10) submetido à hipotermia sistêmica. Dosagens bioquímicas de alanina aminotransferase (ALT), aspartato aminotransferase (AST), bilirrubina direta (BD) e estudo morfológico à microscopia óptica (MO) e microscopia eletrônica de transmissão (MET) foram realizados antes da hipotermia (T0), após a estabilização da temperatura corporal em $10^{\circ} \mathrm{C}$ menor que a temperatura inicial (Test) e após sessenta minutos de hipotermia (T60). Resultados: A dosagem bioquímica das enzimas, aspartato aminotransferase e bilirrubina direta, evidenciaram maiores níveis de lesões no grupo submetido à hipotermia tópica, quando comparado ao grupo de animais submetidos à hipotermia sistêmica. Por sua vez, a dosagem da Alanina aminotransferase não apresentou alterações em ambos os grupos. Tanto a MO quanto a MET, revelou que nos animais do grupo com hipotermia tópica, as lesões hepáticas foram mais intensas quando comparadas ao do grupo com hipotermia sistêmica. Conclusão: A hipotermia tópica é mais lesiva ao fígado do que a sistêmica.
\end{abstract}

Descritores: Anestesia. Hipotermia. Fígado. Cães.

\begin{abstract}
Purpose: To compare hepatic lesions produced by two types of hypothermia; the systemic and the local or topic. Methods: Twenty dogs distributed in two groups were studied: the first submitted to local hypothermia and the second to systemic hypothermia. In all groups, biochemical dosages for alanina allytransferase (A.L.T.), aspartate aminotrasnferase (A.S.T.) and direct bilirubin (T.D.), conventional optical microscopy and electronic transmission microscopy were performed in times T0, Test, and T60, that is, before the hypothermia (T0), after temperature stabilization at $10^{\circ}$ lower than initial temperature (Test), and after sixty minutes of hypothermia (T60). Results: The data analysis, both of the biochemical profile and of the microscopy showed that in the group of animals with selective hypothermia, the hepatic lesions were more intense when compared to the systemic hypothermia group. Conclusion: The selective hypothermia causes more lesions to the liver than the systemic.

Key words: Anesthesia. Hypothermia. Liver. Dogs.

\section{Introdução}

O procedimento terapêutico de Hipotermia considera uma redução da temperatura sanguínea de um animal homeotérmico a níveis inferiores a $35^{\circ} \mathrm{C}$, com a finalidade de reduzir a taxa metabólica basal e o consumo de oxigênio celular, visando a proteção do organismo durante situações

agressivas ou isquemiantes ${ }^{1}$. Nos transplantes, os órgãos são submetidos por um espaço de tempo a baixos níveis de oxigenação e conseqüente isquemia cuja reperfusão desencadeia ou agrava lesões ultraestruturais importantes. Estas lesões teciduais, celulares, endoteliais ou de organelas podem ser reduzidas quando se submete o organismo a hipotermia.
\end{abstract}


Ainda constituem indicações para a hipotermia induzida, as cirurgias intracranianas, sépse, traumatismos encefálicos, grandes queimaduras e cirurgias do parênquima hepático.

As lesões de isquemia e reperfusão no fígado consecutivas aos níveis baixos de oxigenação no processo de coleta e transferência do doador para o receptor são muito estudadas. O mecanismo exato de ação ainda não está bem determinado. Procuram-se situações, drogas e procedimentos que possam atenuar ou abolir suas manifestações. A indução a um estado de hipotermia pode minimizar ou prevenir esta condição ${ }^{2-6}$.

A hipotermia, embora seja técnica antiga e eficaz em determinadas situações, pode provocar, por si só, danos irreversíveis em órgãos submetidos a ela. Foram criados métodos para se obter hipotermia com segurança em cão, que parece ser bom modelo animal de experimentação ${ }^{1}$. Pesquisas com enzimas hepáticas para detecção de lesões, bem como a observação de organelas é de aplicação comum nestes modelos experimentais. Níveis séricos de transaminases como a aspartato aminotransferase (AST) e alanina aminotransferase (ALT) foram estudados, detectando lesões possivelmente causadas pela hipotermia ${ }^{7}$.

Procedimentos de indução a hipotermia requerem cuidados intensivos especiais de monitorização hemodinâmica, respiratória e da temperatura corporal central com ocorrência de importantes alterações do equilíbrio ácido-base e dos níveis de cálcio circulatório que devem ser prontamente identificados e corrigidos ${ }^{8}$. A funções renal, transporte de oxigênio e dióxido de carbono, concentrações de hidrogênio, funções neurológicas centrais e hematológicas sofrem alterações profundas ${ }^{8-10 \text {, }}$.

A hipotermia pode ser aplicada de maneira tópica (superficial ou localizada) ou sistêmica. Ambas, tem suas indicações e contra-indicações bem conhecidas. Entretanto, não foram encontradas na literatura citações de estudos comparativos entre estas duas técnicas e as relações com eventuais lesões hepáticas. Este trabalho tem o objetivo de estudar comparativamente os efeitos da hipotermia sistêmica ou tópica no fígado de cães, por meio da avaliação histológica e funcional hepática.

\section{Métodos}

Todos os procedimentos foram avaliados e aprovados pelo Comitê de Ética em Pesquisa da Universidade Federal de São Paulo -UNIFESP-EPM.

Foram selecionados 20 cães, machos, sem raça definida adultos, com peso médio de $11 \mathrm{Kg}$, provenientes do biotério central da Universidade José do Rosário Vellano UNIFENAS. Permaneceram em gaiolas individuais para aclimatação na ala pré-operatória do centro cirúrgico do Hospital Veterinário por um período de 5 dias, recebendo alimentação ad libitum. Foram distribuídos randomicamente por sorteio em 2 grupos: GI (n=10) com hipotermia tópica e GII(n=10) com hipotermia sistêmica. Foram privados de água por 6 horas e alimentos sólidos por 24 horas antes do experimento. Após a medicação pré-anestésica com 3mg/ $\mathrm{Kg}^{-1}$ de cloridrato de clorpromazina (EV) e indução anestésica com 15mg/ $\mathrm{Kg}^{-1}$ de tiopental sódico (EV), foram entubados e mantidos com ventilação controlada mecânica, sob sistema fechado, vaporizando halotano a 2,5V\%. Após perda do reflexo córneo-palpebral e do estímulo doloroso pela preensão da pata posterior foram estabelecidos a monitorização pela esfignomanometria via Doppler, a pressão venosa central, a oximetria de linha e de pulso, o eletrocardiograma e o eletroencefalograma.

Todos os animais foram submetidos à laparotomia mediana supra-umbilical e exposição do parênquima hepático. Um termômetro de precisão foi inserido, transcutaneamente por contra-abertura no parênquima hepático até a proximidade da veia cava.

Os animais do grupo GI tiveram seus fígados isolados em papel alumínio e envoltos por gelo picado, até que a temperatura, no termômetro atingisse $10^{\circ} \mathrm{C}$ a menos que a temperatura inicial de cada animal $\left(39^{\circ} \mathrm{C}\right)$ caracterizando a hipotermia localizada de condução. Os animais do grupo GII tiveram todo o corpo coberto por sacos de gelo em gel, tendo-se o cuidado de não deixar que o gelo tocasse a cavidade abdominal exposta, até que a temperatura central abaixasse $10^{\circ} \mathrm{C}$ da temperatura inicial $\left(39^{\circ} \mathrm{C}\right)$, caracterizando a hipotermia sistêmica de condução.

Antes da indução da hipotermia, denominado tempo zero (T0), foram coletados $5 \mathrm{ml}$ de sangue com tubo a vácuo, sem anti-coagulantes, pela veia esplênica e armazenados, para a realização das dosagens bioquímicas de AST, ALT e bilirrubina direta (BD) e coletados dois fragmentos do parênquima hepático para análise microscópica óptica (MO) e eletrônica de transmissão (MET).

Após estabilização da temperatura corporal abaixo em $10^{\circ} \mathrm{C}$ da temperatura corporal inicial, denominado tempo de estabilização (Test), procedeu-se a novas coletas de sangue venoso e de fragmentos hepáticos. Decorridos sessenta minutos após atingir a temperatura de estabilização, denominado tempo sessenta (T60), novas amostras de sangue e parênquima hepático foram coletadas. A eutanásia ocorreu pela injeção de cloreto de potássio a 19,1\% em dose suficiente para provocar a parada cardio-respiratória, com animal ainda sob anestesia.

Um dos fragmentos hepáticos em cada tempo da coleta foi processado e corado pela hematoxilina-eosina (HE) para leitura em MO onde analisou-se a presença ou ausência de congestão (edema) intersticial e número de hepatócitos com degeneração hidrópica em 50 campos escolhidos aleatoriamente. O outro fragmento de cada tempo de coleta foi processado para MET e analisado qualitativamente para surpreender alterações de organelas (lisossomos) ou da estrutura do citosol (eletrotransluscência).

O estudo estatístico foi realizado na disciplina de Bioestatística do Departamento de Medicina preventiva da UNIFESP - EPM. De acordo com as variáveis foram aplicados os seguintes testes: 1- Teste “T” de student para comparar o peso dos animais do grupo I ao Grupo II. 2Teste de Mann-Whitney para comparar as variáveis estudadas entre os dois grupos. 3- análise de variância de Friedman para comparar as variáveis nos períodos T0, Test e T60 para os grupos separadamente. Fixou-se em 0,05 ou $5 \%$ (a $£ 0,05$ ) o nível de rejeição da hipótese de nulidade, assinalando-se com um asterisco os valores significantes. 


\section{Resultados}

A análise dos dados coletados revela que os animais submetidos à hipotermia seletiva, alcançaram a temperatura de estabilização em tempo duas vezes menor que os animais submetidos à hipotermia sistêmica (Tabela 1).

TABELA 1 - Cães dos grupos GI e GII segundo o horário inicial da estabilização da temperatura e o tempo decorrido entre os períodos.

\begin{tabular}{llllll}
\hline \multicolumn{2}{c}{ GI } & & \multicolumn{3}{c}{ GII } \\
\hline Inicial & Final & Est-inicial & Inicial & Final & Est-inicial \\
\hline 8:39 & $9: 05$ & 26 & $14: 04$ & $15: 08$ & 64 \\
9:50 & $10: 10$ & 20 & $14: 11$ & $14: 54$ & 43 \\
14:55 & $15: 10$ & 15 & $14: 20$ & $15: 06$ & 46 \\
9:30 & $9: 45$ & 15 & $15: 00$ & $15: 55$ & 55 \\
15:10 & $15: 35$ & 15 & $9: 10$ & $9: 46$ & 36 \\
9:45 & $10: 20$ & 35 & $16: 05$ & $17: 07$ & 62 \\
15:15 & $15: 45$ & 30 & $10: 00$ & $11: 00$ & 60 \\
15:00 & $15: 20$ & 20 & $13: 50$ & $14: 35$ & 45 \\
15:00 & $15: 40$ & 40 & $9: 38$ & $10: 40$ & 62 \\
15:10 & $15: 30$ & 20 & $12: 50$ & $13: 59$ & 69 \\
\hline Média & & $\mathbf{2 3 , 6}$ & & & $\mathbf{5 4 . 2 8}$ \\
& & & & & $*(\mathbf{p}=\mathbf{0 , 0 5})$ \\
\hline
\end{tabular}

\section{Análise da microscopia óptica}

A microscopia de luz convencional evidenciou-se lesões microscópicas bem delimitadas, como degeneração hidrópica e colestase acentuada nos animais de GI, nos tempos Test e T60. Pouca ou nenhuma alteração foi observada ao T0. Entretanto a intensidade de lesão foi significante maior nos animais de GI (Figuras 1, 2, 3), quando comparados aos animais de GII que apenas apresentaram degeneração hidrópica das células hepáticas, evidenciando pouca colestase, pigmentos de bilirrubinas ou outras alterações dignas de notas (Figuras 4, 5 ).

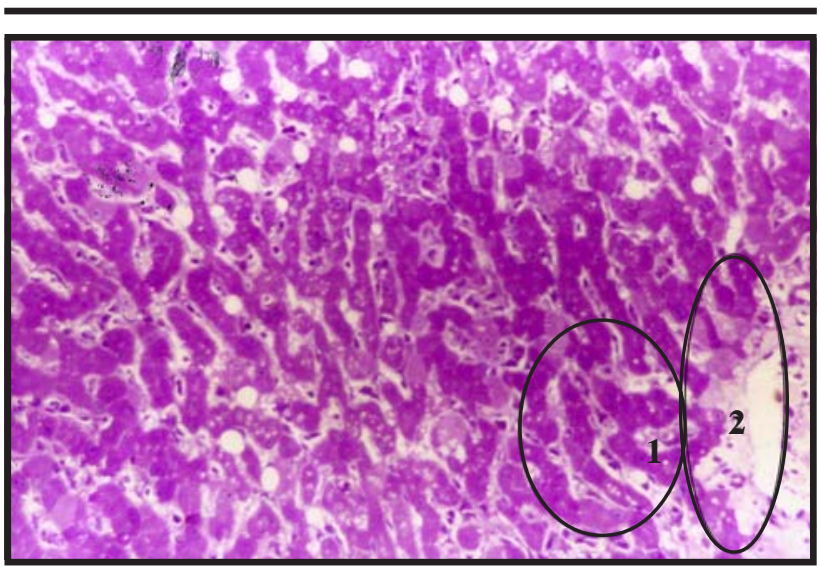

FIGURA 1 - Fotomicrografia de amostra obtida em T0, de animal de GI, evidenciado: em 1 e 2, espaço porta com vênula e arteríola normais, trabéculas ou colunas de hepatócitos em 3. Notar os capilares sinusóides levemente dilatados.(40X-HE)

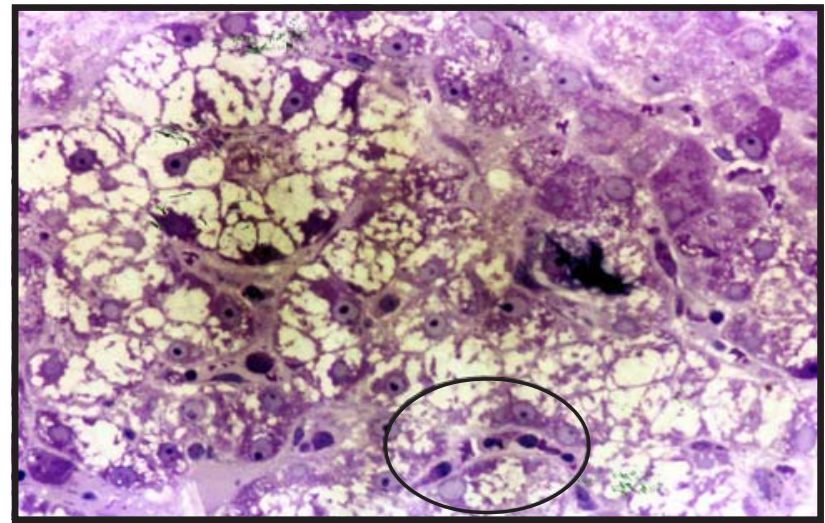

FIGURA 2 - Fotomicrografia de amostra obtida em Test de GI. Notar extensas áreas de degeneração hidrópica em 1, em vacúlo citoplasmático, mantendo-se a integridade do núcleo em 2. (HE-40X)

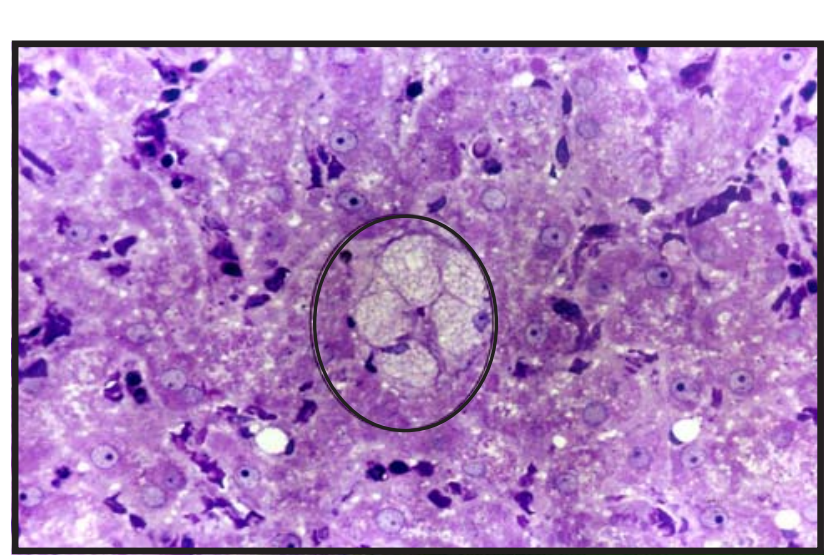

FIGURA 3 - Fotomicrografia de amostra obtida em T60 de GI mostrando extensas áreas de degeneração hidrópica e muitos vacúolos em 1.(HE - 40X)

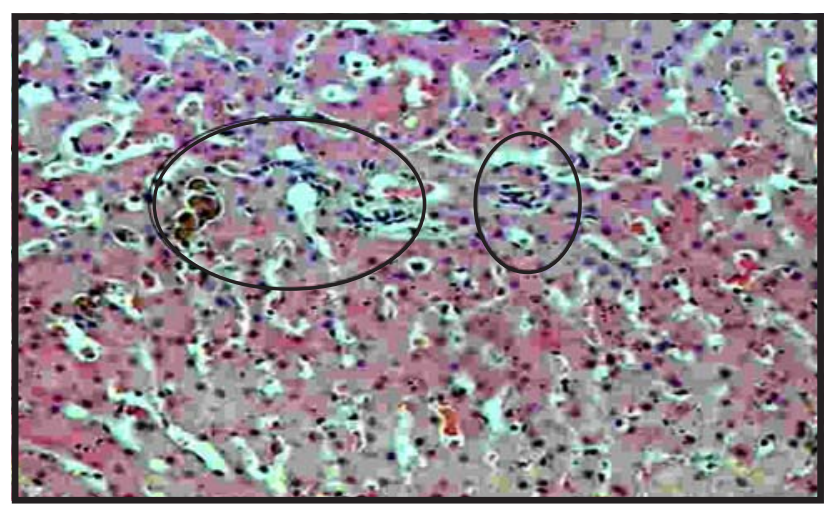

FIGURA 4 - Fotomicrografia de amostra obtida em Test de GII mostrando algumas áreas de colestase e pigmentos de bilirrubina.(HE-40X) 


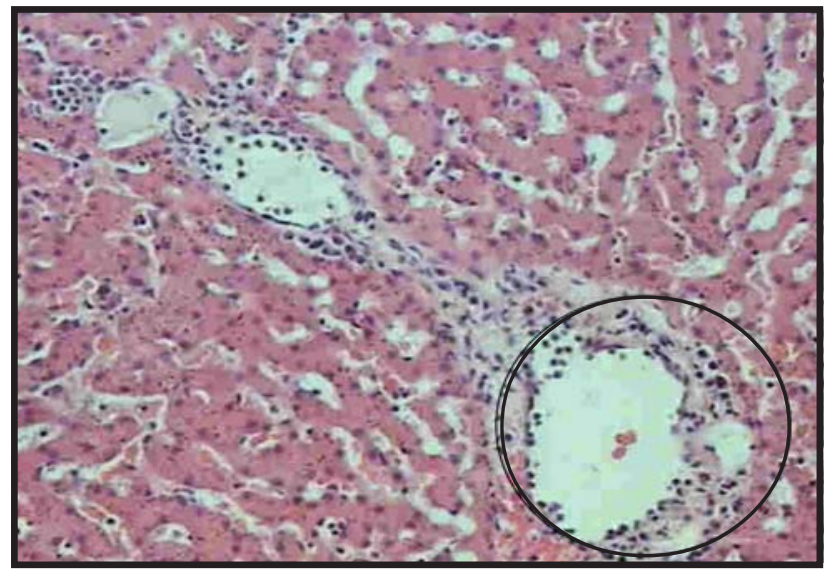

FIGURA 5 - Fotomicrografia em amostra obtida em T60 de GII mostrando veia centro lobular com marginalização e migração neutrofílica. (HE 40X)

\section{Análise ultra-estrutural}

A análise ultra-estrutural das amostras hepáticas dos animais submetidos à hipotermia, nos dois grupos, mostraram em todos os tempos observados (T0, Test e T60), hepatócitos nítidos, poliédricos e certo grau de desorganização de organelas bem evidentes. No entanto, nos tempos Test e T60, houve uma equivalência das alterações ultra-estruturais, que foram muito menos freqüente que no tempo T0 (Figura 6). Os animais de GI, nos tempos Test e T60, apresentaram basicamente as mesmas características evidenciadas em T0, no entanto, chama a atenção o aumento de áreas eletrotransluscentes, demonstrando maior concentração de lesão celular (Figura 7). No grupo GII, observou-se parênquima constituído por hepatócitos bem definidos, mostrando grande concentração de mitocôndrias, retículo endoplasmático granular bastante disperso, pouca concentração de lisossomas e de áreas eletrotransluscentes (Figura 8). Para os animais do grupo GI, nos tempos, Test e T60, houve basicamente as mesmas características, chamando a atenção a grande concentração de áreas eletrotranslucentes, demonstrando maiores níveis de lesões celulares, enquanto que para o grupo GII, as diferenças entre os tempos não foram significativas, com pouca concentração de lisossomos e de mitocôndrias e retículo endoplasmático granular muito disperso, com poucas áreas eletrotranslucentes. A dosagem bioquímica das enzimas, aspartato aminotransferase e bilirrubina direta, evidenciaram maiores níveis de lesões no grupo submetido à hipotermia tópica, quando comparado ao grupo de animais submetidos à hipotermia sistêmica (Tabelas 2 e 4). Por sua vez, a dosagem da Alanina aminotransferase não apresentou alterações em ambos os grupos (Tabela 3).

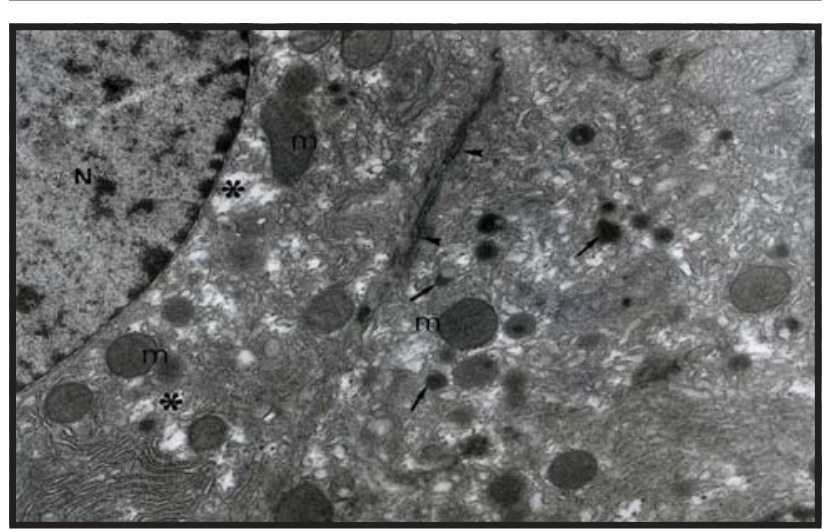

FIGURA 6 - Eletromicrografia de amostra em T0 de GII, mostrando dois hepatócitos interligados por justaposição de membranas citoplasmáticas com adesão celular (cabeça de seta). Notar núcleo evidente, esférico e eucromático $(\mathrm{N})$. Regiões eletrotranslucentes entremeadas no arcabouço de sustentação celular (*), notar os lisossomos de vários tamanhos (setas) e mitocôndrias íntegras (m). (MET 12000X)

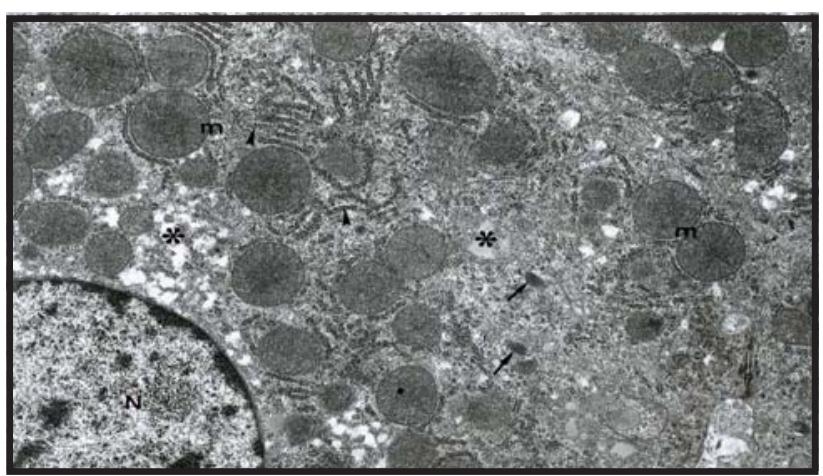

FIGURA 7 - Eletromicrografia de amostra em Test de GI, mostrando um hepatócito com núcleo bastante volumoso e predomínio de eucromatina $(\mathrm{N})$, retículo endoplasmático granular (cabeças de seta), lisossomos (setas), áreas eletrotranslucentes (*), e mitocôndrias integras. (MET 12000X)

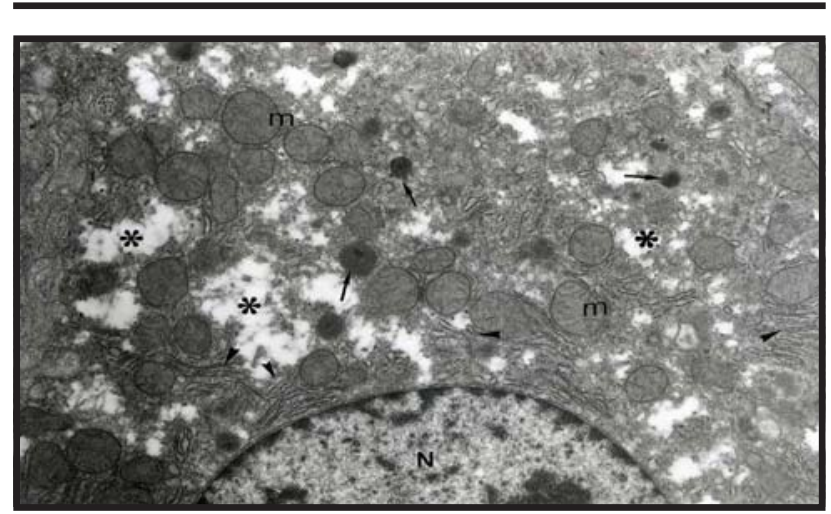

FIGURA 8 - Eletromicrografia de amostra em T60 de GI, evidenciando núcleo bastante volumoso, esférico e eucromático $(\mathrm{N})$, retículo endoplasmático granular (cabeças de seta), lisossomos (setas), grande concentração de espaços eletrotranslucentes $(*)$, e mitocôndrias íntegras (M). (MET 12000X) 
TABELA 2 - Cães do grupo GI e GII submetidos à hipotermia seletiva e sistêmica segundo os valores das dosagens bioquímicas de Aspartato Aminotransferase-A.S.T. (UI/L).

\begin{tabular}{llllll}
\hline & GI & \multicolumn{3}{c}{ GII } \\
\hline T0 & Test & T60 & T0 & Test & T60 \\
\hline 4,20 & 9,64 & 9,64 & 4,2 & 8,75 & 9,76 \\
1,01 & 4,34 & 3,37 & 0,98 & 1,44 & 3,00 \\
2,02 & 4,34 & 39,52 & 2,09 & 3,38 & 4,87 \\
2,21 & 6,75 & 9,64 & 2,34 & 2,00 & 10,9 \\
5,78 & 10,96 & 11,57 & 1,35 & 2,87 & 10,0 \\
2,30 & 9,2 & 8,2 & 2,30 & 2,09 & 2,98 \\
1,29 & 8,2 & 90,0 & 3,30 & 3,50 & 4,44 \\
3,45 & 20,24 & 26,51 & 1,09 & 6,87 & 4,98 \\
10,02 & 11,57 & 10,60 & 9,80 & 8,44 & 11,00 \\
21,03 & 5,78 & 13,98 & 20,00 & 5,87 & 5,07 \\
\hline $\mathbf{4 , 8 6}$ & $\mathbf{9 , 1}$ & $\mathbf{2 2 , 0 3}$ & $\mathbf{4 , 7 4}$ & $\mathbf{4 , 5 2}$ & $\mathbf{6 , 7}$ \\
\hline \multicolumn{5}{c}{ T60*(GI> $\mathbf{G I I )}$} \\
\end{tabular}

TABELA 3 - Cães dos grupos GI e GII submetidos à hipotermia seletiva e sistêmica, respectivamente, segundo os valores das dosagens bioquímicas de alanina aminotransferase - A.L.T. (UI/L).

\begin{tabular}{llllll}
\hline \multicolumn{3}{l}{ G } & \multicolumn{3}{c}{ GII } \\
\hline TO & Test & T60 & T0 & Test & T60 \\
\hline 5,3 & 10,48 & 1,44 & 2,21 & 4,35 & 7,70 \\
0,48 & 1,44 & 3,86 & 2,90 & 2,09 & 2,09 \\
1,44 & 1,44 & 0,48 & 2,09 & 2,38 & 1,07 \\
4,0 & 3,0 & 26,0 & 3,09 & 1,40 & 3.09 \\
3,85 & 0,48 & 10,12 & 3,98 & 8,07 & 8,0 \\
5,30 & 2,01 & 0,00 & 2,70 & 6,00 & 6,09 \\
5,30 & 0,50 & 1,44 & 4,09 & 3,70 & 8,64 \\
4,94 & 7,71 & 7,71 & 4,09 & 7,09 & 5,09 \\
2,80 & 1,44 & 11,57 & 2,09 & 4,74 & 3,00 \\
4,35 & 6,26 & 5,30 & $\mathbf{4 , 0 9}$ & 5,87 & 5,09 \\
\hline $\mathbf{3 , 7 7}$ & $\mathbf{4 , 4 8}$ & $\mathbf{6 , 7 9}$ & $\mathbf{3 , 1 3}$ & $\mathbf{4 , 5 7}$ & $\mathbf{4 , 9 8}$ \\
\hline
\end{tabular}

Análise de variância de Friedman

$$
\text { (GI=GII) }
$$

TABELA 4 - Cães dos grupos GI e GII, respectivamente, segundo os valores das dosagens de Bilirrubina Direta(BD) UI/L

\begin{tabular}{llllll}
\hline & G & & \multicolumn{3}{c}{ GII } \\
\hline T0 & Test & T60 & T0 & Test & T60 \\
\hline 0,3 & 0,65 & 0,54 & 0,2 & 0,2 & 0,3 \\
0,05 & 0,65 & 0,76 & 0,1 & 0,3 & 0,2 \\
0,12 & 0,31 & 0,60 & 0,12 & 0,23 & 0,21 \\
0,20 & 0,32 & 0,32 & 0,11 & 0,21 & 0,11 \\
0,11 & 0,21 & 0,30 & 0,2 & 0,32 & 0,21 \\
0,20 & 0,76 & 0,65 & 0,34 & 0,21 & 0,23 \\
0,37 & 0,65 & 0,87 & 0,4 & 0,31 & 0,34
\end{tabular}

\begin{tabular}{lccccc}
0,1 & 0,32 & 0,21 & 0,1 & 0,1 & 0,13 \\
0,1 & 0,43 & 0,45 & 0,21 & 0,4 & 0,32 \\
0,20 & 0,53 & 0,50 & 0,21 & 0,23 & 0,20 \\
\hline $\mathbf{0 , 2 1}$ & $\mathbf{0 , 4 7}$ & $\mathbf{0 , 5 2}$ & $\mathbf{0 , 1 9}$ & $\mathbf{0 , 2 5}$ & $\mathbf{0 , 2 5}$ \\
\hline \multicolumn{5}{c}{ Teste de Mann-Whitney } \\
Test $=\mathbf{1 2 , 5 *}$ (GI > GII) \\
T60 = 6,5* $\mathbf{~ ( G I ~ > ~ G I I ) ~}$
\end{tabular}

TABELA 5 - Médias das contagens de hepatócitos com degeneração hidrópica à microscopia óptica (HE - 40X), nos grupos I e II e nos tempos T0, Test e T60.

\begin{tabular}{llllll}
\hline \multicolumn{1}{c}{ GI } & \multicolumn{3}{c}{ GII } \\
\hline T0 & Test & T60 & T0 & Test & T60 \\
\hline 25,3 & 55,8 & 52,0 & 23,4 & 42,0 & 45,9 \\
16,02 & 30,6 & 44,2 & 15,0 & 17,1 & 26,1 \\
15,10 & 52,1 & 74,2 & 14,0 & 28,3 & 32,3 \\
09,03 & 51,2 & 50,6 & 10,5 & 12,7 & 11,9 \\
19,03 & 70,4 & 68,6 & 18,0 & 45,0 & 51,0 \\
28,05 & 66,5 & 66,4 & 29,0 & 37,0 & 40,7 \\
30,04 & 54,8 & 62,5 & 31,7 & 39,0 & 11,8 \\
25,06 & 63,7 & 85,8 & 24,6 & 28,9 & 24,9 \\
21,04 & 60,3 & 66,5 & 22,6 & 21,7 & 25,2 \\
31,09 & 57,8 & 65,2 & 33,2 & 40,5 & 37,2 \\
\hline $\mathbf{2 2 , 9}$ & $\mathbf{5 6 , 3}$ & $\mathbf{6 3 , 6}$ & $\mathbf{2 2 , 2}$ & $\mathbf{3 1 , 2 8}$ & $\mathbf{3 0 , 7 0}$ \\
\hline \multicolumn{5}{c}{ TO $<$ Test* e T60* } \\
\end{tabular}

\section{Discussão}

Para caracterizar a hipotermia a temperatura corpórea estabelecida para a espécie humana deve ser inferior a $35^{\circ} \mathrm{C}$. Em cães, devido a diferenciação interespécies e tamanho corporal, este valor ainda não está bem estabelecido ${ }^{11,12,13,14,15}$. Na dependência do tipo de técnica utilizada e das necessidades do procedimento, pode-se obter diferentes graus de hipotermia: leve é quando se mantém temperatura ao redor de $35^{\circ} \mathrm{C}$, moderada quando está na faixa de 28 a $34^{\circ} \mathrm{C}$ e profunda em temperaturas inferiores a $28^{\circ} \mathrm{C}$. No modelo proposto neste experimento reduziu-se à temperatura corporal dos animais em $10^{\circ} \mathrm{C}$ dos valores iniciais registrados para cada animal, para caracterizar com segurança uma hipotermia profunda ${ }^{16}$.

Foram escolhidos cães, machos, adultos com média de peso geral de $10,57 \mathrm{Kg}$, sendo de $11,58 \mathrm{Kg}$ para o grupo GI e 9,92 kg para o grupo GII. O teste “t” de Student não demonstrou alterações significativas entre estas médias, caracterizando a homogeneidade da amostra.

Os procedimentos de anestesia e monitorização foram escolhidos levando-se em conta que as drogas utilizadas pouco influenciariam na função hepática e auxiliariam na perda da temperatura ${ }^{17,18,19}$. Uma vez que os procedimentos foram semelhantes em ambos os grupos, as eventuais interferências foram propiciadas a ambos indistintamente.

Procedimentos de monitorização são de extrema importância nos animais submetidos a hipotermia. 
Alterações hemodinâmicas, hemogasométricas, nos níveis de eletrólitos séricos ou na condução elétrica intrínseca cardíacas podem levar à condições que interferem de modo acentuado nos próprios parâmetros que estão em pesquisa ${ }^{8,9,10,4}$. A observação constante dos dados monitorados pelo anestesista possibilitaram a manutenção das atividades vitais do animal de modo a dar ensejo a uma padronização dos procedimentos de coleta de dados sem que se possa atribuir um agravamento pelas condições adversas proporcionadas pelas alterações anteriormente elencadas.

Os animais submetidos a hipotermia seletiva ou tópica (GI) atingiram a temperatura de $10^{\circ} \mathrm{C}$ abaixo da temperatura corporal inicial com média de 23,6 minutos, tempo significantemente menor que os animais submetidos a hipotermia sistêmica (GII) cuja média foi de 54,2 minutos (Tabela 1). É evidente que a massa hepática, diretamente em contato com o agente de resfriamento (gelo gel), sendo menor que a massa corporal do animal como um todo, resfriasse em menor tempo. Também existiu a dificuldade de envolvimento de toda massa corporal do animal devido a sua conformidade anatômica e posicionamento na mesa operatória.

Este resfriamento mais rápido parece ter uma influência definitiva na integridade do parênquima hepático e também na integridade de sua máquina enzimática. Os animais do resfriamento tópico mostraram-se mais susceptíveis a alterações morfológicas (MO e MET) e nas dosagens das enzimas hepáticas que estão associadas a alterações da viabilidade celular.

Os valores de AST, ALT e BD apresentaram variações individuais importantes já no tempo zero onde não havia nenhum fator de agressão importante a não ser o trauma anestésico e operatório e o envolvimento hepático ou corporal pelo gelo (Tabelas 2, 3 e 4). Essa variação foi observada também na literatura pertinente para esta espécie animal (cão). Nesta espécie, dentre os fatores que influenciam nas variáveis do perfil enzimático estudado, estão a idade dos animais, falta de homogeneidade da raça, peso, dieta e método específico de coleta. As enzimas refletem o estado metabólico do corpo, sob condições de anaerobiose, em coleta de órgãos, pode haver incremento considerável da taxa de enzimática ${ }^{19}$. Apesar desta variação individual os testes estatísticos trabalhando com as médias encontradas permitiram a análise e comparação dos grupos, pois houve uma clara alteração de comportamento destas médias nos tempos de observação considerados.

Fujii $^{12}$ correlacionou a necrose hepatocística, hepatite e a colestase ao aumento das taxas de AST, ALT e gama glutamiltranspeptidase (GTP). Os parâmetros considerados são expressivos das lesões hepáticas conseqüentes a agressões variadas (mecânicas, metabólicas ou infecciosas).

A alteração da AST (Tabela 2) mostra de modo mais evidente um aumento significativo entre os tempos Test e T60 nos animais com hipotermia tópica sendo bem menor nos animais da hipotermia sistêmica. A ALT manteve a mesma tendência (Tabela 3 ), porém de modo menos evidente. A BD teve também um aumento significante entre Test e T60 nos animais de hipotermia tópica (Tabela 4) sendo que nos animais da hipotermia sistêmica as achados foram menos evidentes. Acrescente-se ao quadro enzimático uma correspondente e paralela alteração da morfologia tecidual, tanto na microscopia óptica, quanto na microscopia eletrônica de transmissão, nos grupos e tempos estudados.

Os resultados obtidos permitem inferir que a hipotermia em si é um fator agressivo ao tecido hepático e leva a alterações morfológicas teciduais e do perfil enzimático hepático. O desdobramento e influência destas alterações nos eventos futuros de um transplante, ou seja, em órgão submetido a isquemia/reperfusão é uma questão em aberto. O modelo proposto mostrou-se promissor para o aprofundamento do estudo da hipotermia não só na diferenciação em tópica e sistêmica, mas também na possibilidade de avaliar parâmetros mais sensíveis e específicos da atividade e morfologia hepática.

\section{Conclusão}

A hipotermia hepática tópica provoca lesões teciduais e funcionais do hepatócito mais intensas do que a hipotermia sistêmica.

\section{Referências}

1. Lumb WW, Jones WE. Other methods for producing general anesthesia. In: Veterinary Anesthesia. Philadelphia, Lea \& Fabiger, 1973;319-42.

2. Northway RB. A techinic for open heart surgery using hypothermia. Small An Clinical Med. 1969;10(2):875-9.

3. Lee JA, Atkinson RS. Hipotermia induzida. In: Manual de anestesiologia. Rio de Janeiro, Atheneu, 1976;24:561-72.

4. Moraes NA. Efeitos da hipotermia de superfície sobre o equilíbrio ácido-básico e eletrolítico em cães. Tese de Mestrado, UFSM, 1984.

5. Bruckberg GD, Brasier Jr , Nelson RL. Studies of the effects of hypothermia on regional miocardial blood flow and metabolism during cardiopulmonary bypass. J Thorac Cardiovasc Surg. 1977;73:87.

6. Chitwood WR, Sink JD, Hill RG. The effects of hypothermia on myocardial consumption and transmural coronary blood flow in the potassium arrested heart. Ann Surg. 1979;190:106-16.

7. Takahashi T, Morishita Y, Ichikawa H, Sato Y, Suzuki M, Ohya T, Tomisawa N. Multiple organ harvesting from a single donor for transplantation. A comparision of simple cooling technique and bypass technique. J Cardiovasc Surg. 1999;40(3):347-53.

8. Piccioni MA, Auler Jr JOC. Equilíbrio ácido Base durante a hipotermia. Rev Bras Anest. 1992;42(4):297-63.

9. Gomes OM, Amaral RVC. Hipotermia em cirurgia cardíaca. In: Zerbine EJ. Clinica Cirurgica Alípio Correa Netto. São Paulo, Savier, 1974;43:432-4.

10. Mohri H, Dillard DH, Crawford EW, Martin WE, Merendino RA. Method of surface-induced deep hypothermia for open heart surgery in infants. J Thoracic Cardiovasc Surg.St Louis, 1969;58(2):262-70.

11. Allen, FM. Resistance of periferal tissues to asphyxia at various temperatures. Surg Gynecol Obstet. 1938;67:746.

12. Fuji T. Toxicological correlation between changes in blood biochemistry parameters and liver histopathological finding. J Toxicol Sci. 1997:22(3):161-83. 
13. Blue JT. Short CE. Preanesthetic avaluation and clinical pathology. In: Short CE. Principles and practice of Veterinary Anesthesia. Baltimore: Williams \& Wilkins. 1987; 3:7.

14. Imre MM, Hall T. Body temperature and anaesthesia. $\mathrm{Br}$ J Anaesth. 1990;64:346.

15. Hall LW, Lehman H, Silk E. Response in dogs to relaxants derived from succinin acid and choline. Br Med J. 1953;1:134.

16. Klebanoff G, Hollander D, Cosin BA. Sanguineous hypothermia total body washout in treatment of stage
IV hepatic coma. J Surg Res. 1972;12:1

17. Haskins SC. Hypothermia and its preventions during general anesthesia in cats. Am Vet Res. 1981;42:856-61.

18. Rawling CA, Kolata RJ. Cardiopulmonary effects os thiopental/lidocaína combination during anesthetic induction in the dog. Am J Vet Res. 1983;44:144.

19. Asano M, Inoue K, Ando s, Bito A, Shiojiri Y, Yamada M, Takada T. Optimal temperature of ocntinous lodocaine perfusion for the heart preservation. Jpn J Thorac Cardiovasc Surg. 2003;51(1):1-9.
Correspondência:

Murched Omar Taha

Rua Botucatu 740

04023-900 São Paulo - SP

Tel: (11)5576-4272 / Fax (11) 5571-0233

taha@uol.com.br
Conflito de interesse: nenhum Fonte de financiamento: nenhuma

Recebimento: 23/11/2004

Revisão: 21/12/2004

Aprovação: 20/011/2005

\section{Como citar este artigo:}

Siqueira VJ, Taha MO, Fagundes DJ, Juliano Y,Gomes PO, Bruzadelli RMZ, Caputto LG. Functional and morphological study of the local and systemic hypothermia on dogs' liver. Acta Cir Bras [serial online] 2005 Mar-Abr; 20(2). Disponível em URL: http://www.scielo.br/acb

Figuras coloridas disponíveis em http://www.scielo.br/acb 